\title{
Tidally-triggered disk thickening. I. Observations
}

\author{
V. Reshetnikov ${ }^{1}$ and F. Combes ${ }^{2}$ \\ 1 Astronomical Institute of St. Petersburg State University, 198904 St. Petersburg, Russia \\ 2 DEMIRM, Observatoire de Paris, 61 Av. de l'Observatoire, F-75014 Paris, France
}

Received July 18; accepted October 3, 1995

\begin{abstract}
As part of an investigation on what effect galaxy interactions have on the $z$-structure of the disks of involved galaxies, we present here new photometric data in the $B, V, I$ passbands for a sample of 24 interacting systems and 7 non-interacting galaxies. Isophotes, total magnitudes and colours of all sample galaxies (67) are determined. Our estimates and the comparison with the published results show that the accuracy of our photometry is about 0.15 mag.
\end{abstract}

Key words: galaxies: interactions; photometry; spiral

\section{Introduction}

External perturbations due to galaxy interactions have often been invoked to explain the presence of thick disks in spiral galaxies (Binney \& Tremaine 1987 and references therein). Forces perpendicular to the plane can excite $z$ oscillations and warps, and through dynamical friction, the relative orbital energy of the satellite is lost while the target disk is heated. The efficiency of this process is not clearly known. Analytical estimations and numerical simulations have shown that the efficiency depends on various parameters, as for instance the ratio of dark to visible matter, and the fraction of total mass in the disk. Another phenomenon can moderate the disk thickening during interaction and accretion of a companion: the gas accreted can, by its dissipative character, settle in a thin plane, and make the disk thinner by its direct gravitational effect, and by the subsequent star formation.

Besides, galactic disks can also be thickened by indirect processes: it has been shown for instance that tidal interactions favor the formation of bars (Noguchi 1987; Gerin et al. 1990). Barred galaxies are more frequently seen in groups or in binaries (Thompson 1981; Elmegreen \& Elmegreen 1982). The presence of bars and the implied vertical resonances can excite a peanut-shape or bending mode, that can produce a very thick disk (Combes et al. 1990; Raha et al. 1991).

The efficiency of tidal thickening could set stringent limits on the current rate of galaxy merging and galaxy infall. According to Toth \& Ostriker (1992), the thick-

Send offprint requests to: F. Combes ness of the Milky Way disk implies that no more than $4 \%$ of its mass can have accreted within the last $510^{9} \mathrm{yr}$. They claim that the currently fashionable theory of structure growth by hierarchical merging is not supported by the presence of thin galactic disks, cold enough for spiral waves to develop. This argument is supported by the recent simulations of Quinn et al. (1993) that show that a single merger is sufficient to destroy a thin disk, even if the mass of the satellite is only a few percent that of the disk. However, their calculations did not include any dissipative component, or gas accretion, that could modify the results.

From the observational standpoint this problem is not investigated entirely. Our preliminary consideration (Reshetnikov et al. 1993a) have shown that the disks of strongly interacting spirals are 2-3 times thicker as compared with the disks of normal spiral galaxies. But the small number of objects in our sample (only 6 galaxies) did not permit to study this question in details.

In the present project we plan to investigate the efficiency of tidal disk thickening by observing the thickness of planes in a large enough sample of edge-on interacting galaxies, in comparison to a control sample of isolated galaxies. In this article we present our sample, the observations and general photometric results. A detailed discussion of the results, together with luminosity profiles, will be given in a forthcoming paper. 


\section{Observations and data reductions}

The sample for this study was selected from the atlases by Arp (1966), Vorontsov-Velyaminov (1977) and from the Catalogue of isolated pairs (Karachentsev 1972, 1987). We chose to study strongly interacting systems with apparently edge-on spiral galaxies. Due to limited field size, we selected systems with an apparent angular diameter of $\lesssim 3^{\prime}$. We included 24 interacting systems (mainly double) in our final sample (see Table 1 and Fig. 1). The sample is relatively complete. We included almost all interacting systems from the above catalogs that were suitable in angular diameter and could be observed during our observational run. As it is apparent in Fig. 1, the sample includes a wide morphological range of interacting edge-on galaxies, from early- to late-type spirals. As the control sample we will consider literature data as well as our own observations of 7 nearly edge-on non-interacting galaxies from UGC (Nilson 1973).

\subsection{Data acquisition}

The data were acquired during 19-23 July 1993 with the OHP $1.2 \mathrm{~m}$ telescope at the $f / 6$ Newton focus. The detector was a TK512 CCD of $512 \times 512$ square pixels. The scale was $0 . \prime 75$ pixel $^{-1}$, giving a field of view of $\approx 6 ! 4 \times 6 ! 4$. This device has $10.1 \mathrm{e}^{-} /$pixel readout noise and a conversion factor of $6.5 \mathrm{e}^{-} / \mathrm{ADU}$. The used passbands were Johnson $B, V$ and Cousins $I$. The seeing during our observations was not good $\left(\approx 2-4^{\prime \prime}\right)$. A log of observations is given in Table 1.

Photometric calibration for four nights (from July 20 to July 23) was accomplished using repeated observations of the $B V I$ standard stars from Landolt (1983) and Smith et al. (1991). Care was taken to ensure that the colours of standard stars matched the range of galaxian colours. We used the following extinction coefficients for the OHP: $0 .{ }^{\mathrm{m}} 34,0 . \mathrm{m} 19$, and 0.08 in $B, V$ and $I$ passbands correspondingly (Chevalier \& Ilovaisky 1991). The derived transformations (mean for four nights) from our "outside atmosphere" instrumental magnitudes to the standard BVI system are:

$$
\begin{gathered}
B-b(1 \mathrm{~s})=0.173(b-v)+20.96, \\
B-V=1.090(b-v)-0.615, \\
V-I=1.109(v-i)+0.90 .
\end{gathered}
$$

Standard stars follow the above equations with rms residuals of $0 .{ }^{\mathrm{m}} 02-0{ }^{\mathrm{m}} 03$.

Weather conditions during the first night of observations (19/20 July) were non-photometric. But since the main goal of our work was to obtain the surface brightness distributions for the largest possible sample of edgeon galaxies, we kept also these observations. To calibrate these frames (Arp 30, Arp 124, Arp 150, Arp 278, K 540, $\mathrm{K} 547$ ) we made observations of these galaxies with short exposures (about $1 \mathrm{~min}$ ) during the next four nights. Then, using short-exposure images obtained in photometric conditions, we found individual transformation equations for each object observed in the first night. Naturally, such a second-order calibration gives a worse accuracy for the zero point of the magnitude scale for these objects

\begin{tabular}{|c|c|c|c|c|c|}
\hline \multirow[t]{2}{*}{ Object } & \multirow[t]{2}{*}{ Data } & \multicolumn{3}{|c|}{ Integration time (min) } & \multirow[t]{2}{*}{$\mu_{V}$} \\
\hline & & $B$ & $V$ & $I$ & \\
\hline Arp 30 & Jul/19/93 & 20 & 10 & 10 & 24.9 \\
\hline Arp 71 & $\mathrm{Jul} / 22 / 93$ & 10 & 5 & 5 & 24.7 \\
\hline Arp 112 & $\mathrm{Jul} / 21 / 93$ & 15 & 5 & 5 & 24.7 \\
\hline Arp 121 & $\mathrm{Jul} / 23 / 93$ & 5 & 3 & 3 & 24.0 \\
\hline Arp 124 & Jul/19/93 & 15 & 5 & 5 & 23.4 \\
\hline Arp 127 & $\mathrm{Jul} / 23 / 93$ & 10 & 5 & 5 & 24.4 \\
\hline Arp 150 & $\mathrm{Jul} / 19 / 93$ & 15 & 7 & 7 & 25.0 \\
\hline Arp 208 & $\mathrm{Jul} / 23 / 93$ & 15 & 7 & 7 & 24.9 \\
\hline Arp 242 & $\mathrm{Jul} / 22 / 93$ & 10 & 5 & 3 & 24.5 \\
\hline Arp 278 & Jul/19/93 & 10 & 5 & 7 & 25.0 \\
\hline Arp 284 & $\mathrm{Jul} / 20 / 93$ & - & 5 & 5 & 24.4 \\
\hline Arp 286 & $\mathrm{Jul} / 23 / 93$ & 10 & 5 & 5 & 24.0 \\
\hline Arp 295 & $\mathrm{Jul} / 21 / 93$ & 15 & 5 & 5 & 24.6 \\
\hline VV 426 & Jul/20/93 & 20 & 10 & 10 & 25.4 \\
\hline VV 489 & $\mathrm{Jul} / 22 / 93$ & 15 & 7 & 7 & 25.2 \\
\hline VV 490 & $\mathrm{Jul} / 23 / 93$ & 10 & 5 & 5 & 24.3 \\
\hline VV 679 & $\mathrm{Jul} / 20 / 93$ & 15 & 5 & 5 & 24.4 \\
\hline VV 773 & $\mathrm{Jul} / 20 / 93$ & 15 & 5 & 5 & 24.4 \\
\hline K 3 & $\mathrm{Jul} / 21 / 93$ & 10 & 5 & 5 & 24.6 \\
\hline K 10 & $\mathrm{Jul} / 22 / 93$ & 10 & 5 & 5 & 24.6 \\
\hline K 14 & $\mathrm{Jul} / 22 / 93$ & 10 & 5 & 5 & 24.6 \\
\hline K 540 & $\mathrm{Jul} / 19 / 93$ & 15 & 7 & 7 & 24.8 \\
\hline K 547 & Jul/19/93 & 15 & 7 & 7 & 25.0 \\
\hline K 585 & $\mathrm{Jul} / 20 / 93$ & 15 & 5 & 5 & 24.6 \\
\hline UGC 11132 & $\mathrm{Jul} / 22 / 93$ & 15 & 5 & 5 & 24.7 \\
\hline UGC 11230 & $\mathrm{Jul} / 21 / 93$ & 15 & 5 & 5 & 24.8 \\
\hline UGC 11301 & $\mathrm{Jul} / 21 / 93$ & 15 & 5 & 5 & 23.8 \\
\hline UGC 11838 & $\mathrm{Jul} / 20 / 93$ & 15 & 5 & 5 & 25.0 \\
\hline UGC 11841 & $\mathrm{Jul} / 23 / 93$ & 15 & 7 & 7 & 24.8 \\
\hline UGC 11859 & $\mathrm{Jul} / 22 / 93$ & 15 & 7 & 7 & 24.9 \\
\hline UGC 11994 & $\mathrm{Jul} / 23 / 93$ & 7 & 3 & 3 & 24.3 \\
\hline
\end{tabular}
(about $\pm 0 . \mathrm{m} 10$ ).

Table 1. Observations

\subsection{Data reduction}

The photometry was performed using the MIDAS software package at the Meudon Observatory. The primary reduction included the standard steps: bias subtraction, flat fielding with dome flats, sky subtraction, and removal of the matrix defects. In general the diameters of galaxies in our sample are quite small $\left(\approx 1^{\prime}-2^{\prime}\right)$ with respect to the field size, allowing a careful sampling of the sky 
Arp 30

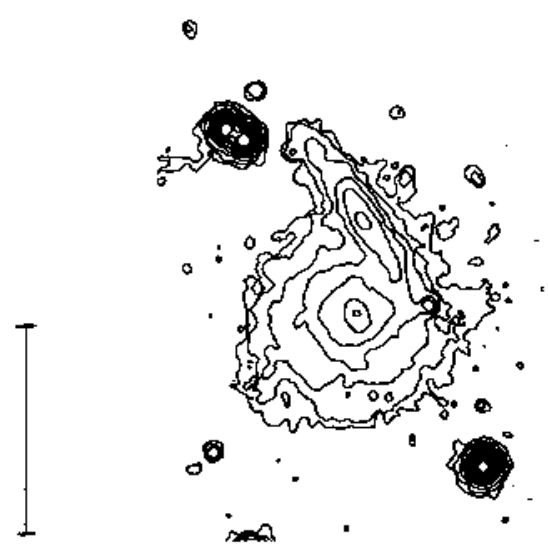

$\operatorname{Arp} 71$

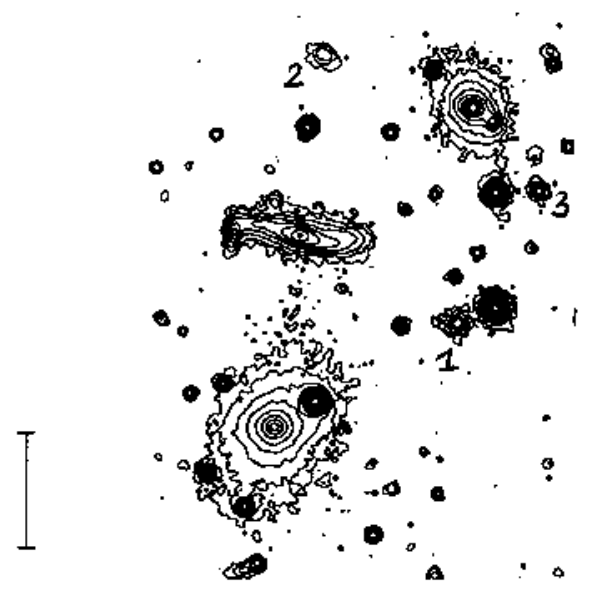

$\operatorname{Arp} 112$

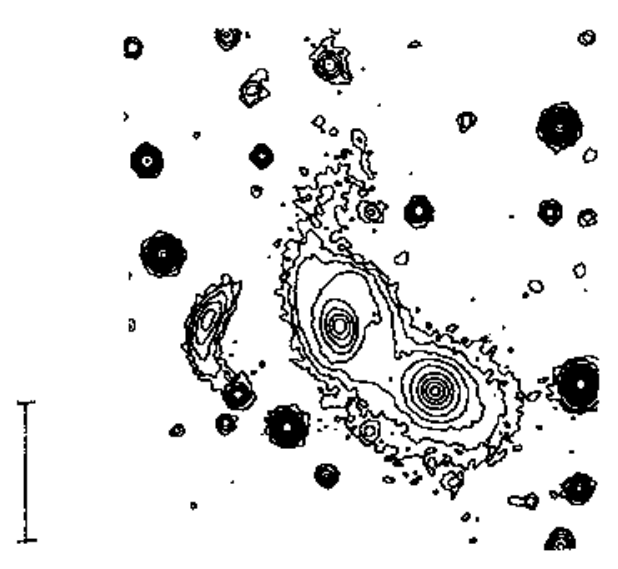

Arp 121

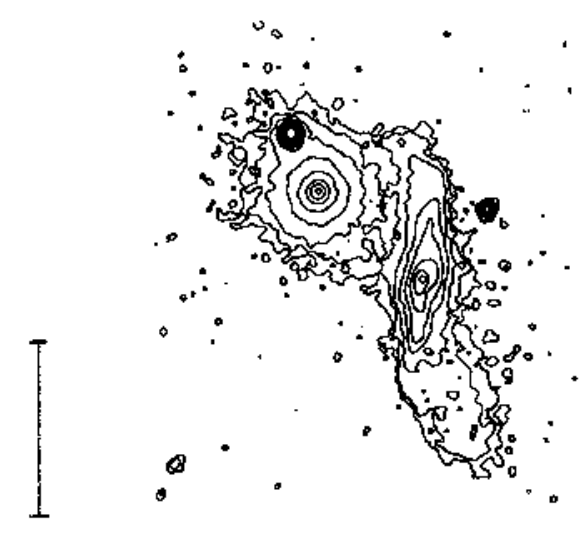

$\operatorname{Arp} 124$

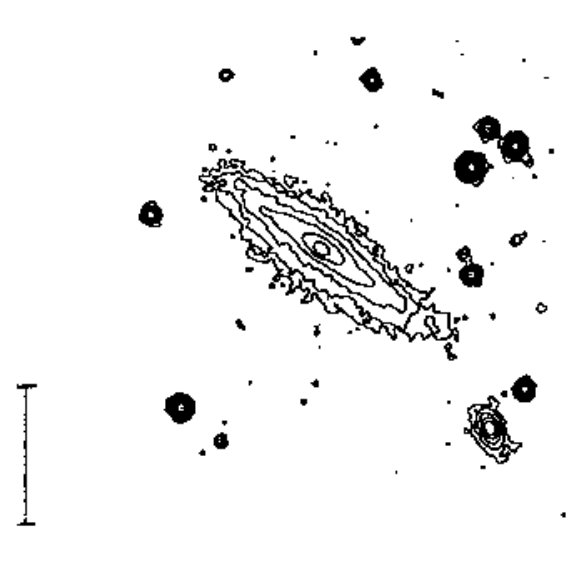

$\operatorname{Arp} 127$

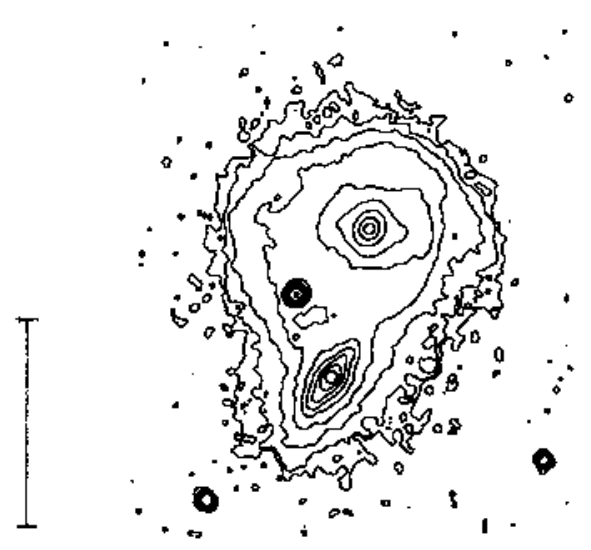

Fig. 1. $V$ band isophotal contour images of the sample galaxies. The isophotes are separated by 0.75 mag, the faintest contours are listed in Table 1. North is at the top, East at the left. The bar length is 1 arcmin 


\section{Arp 150}

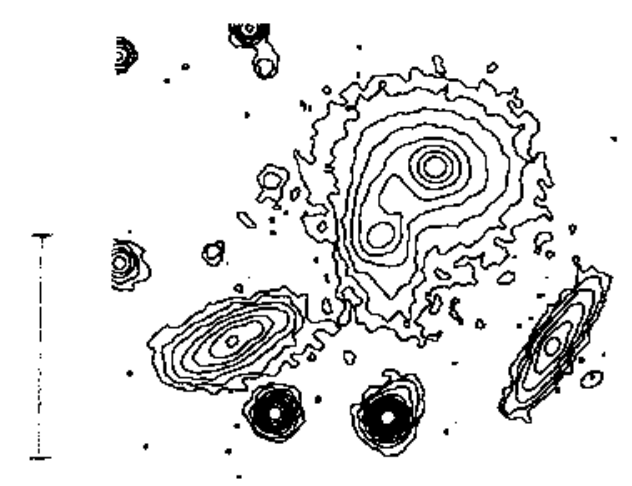

Arp 208

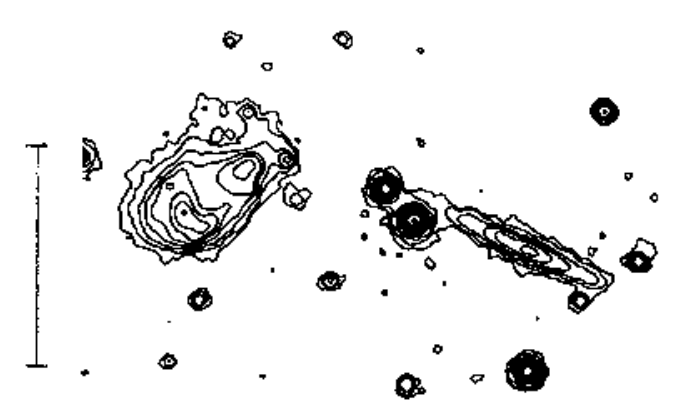

Arp 242

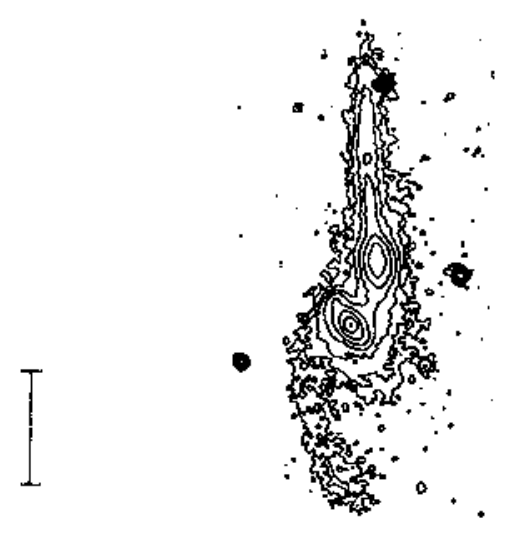

$\operatorname{Arp} 278$

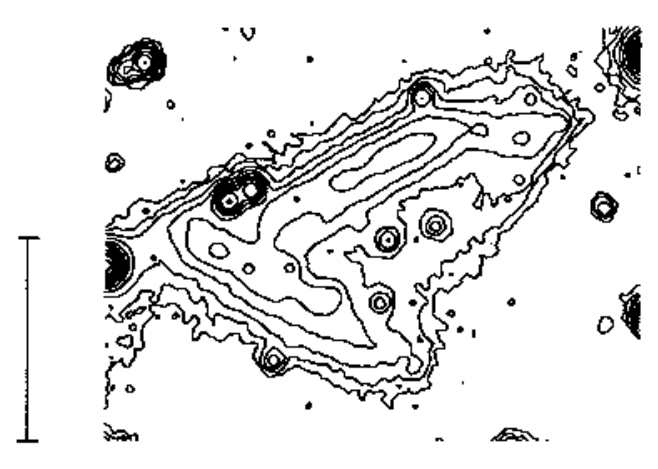

Arp 284

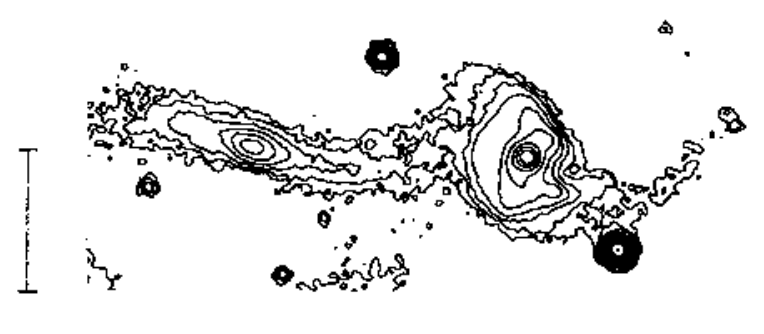

Arp 286

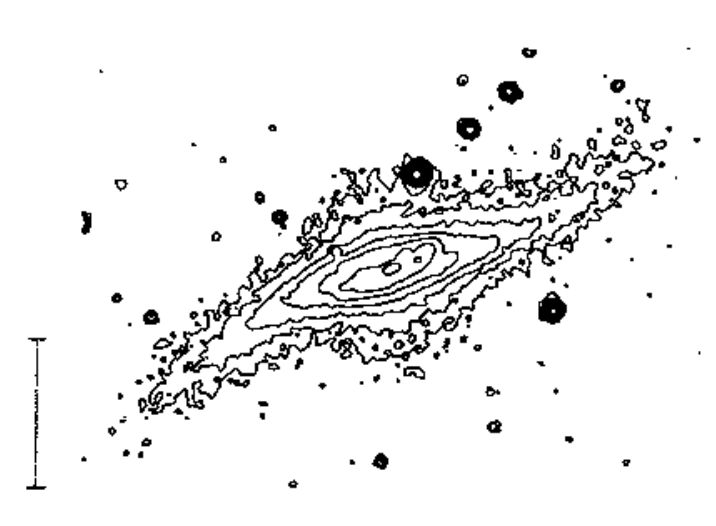

Fig. 1. continued 


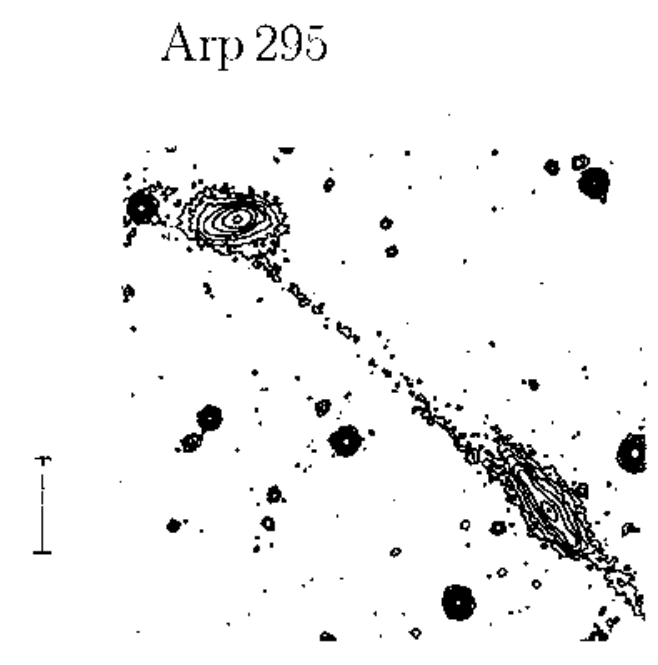

VV 426

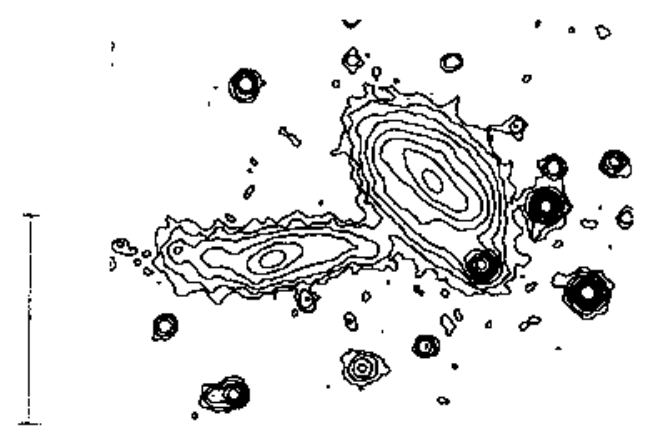

VV 489

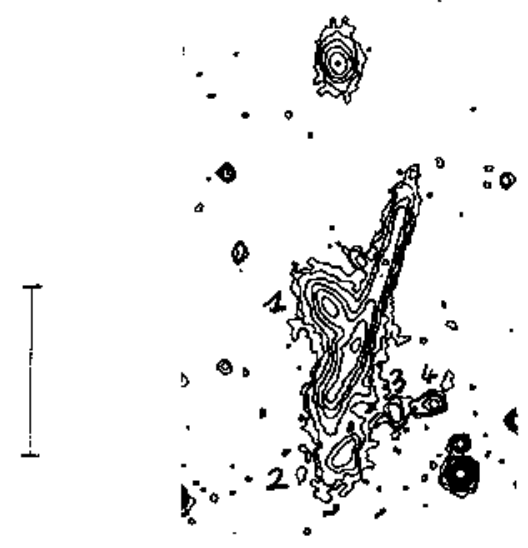

VV 490

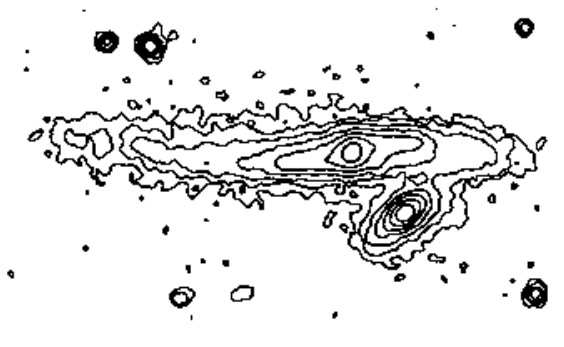

VV 679

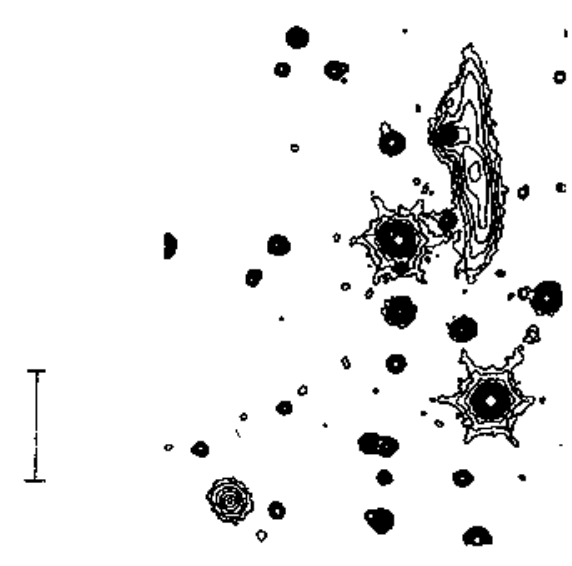

VV 773

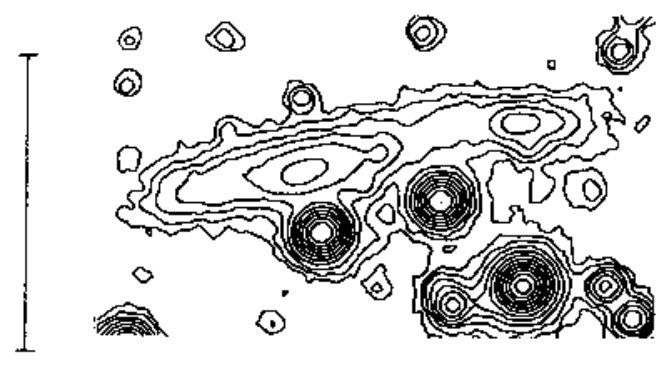

Fig. 1. continued 
K 3

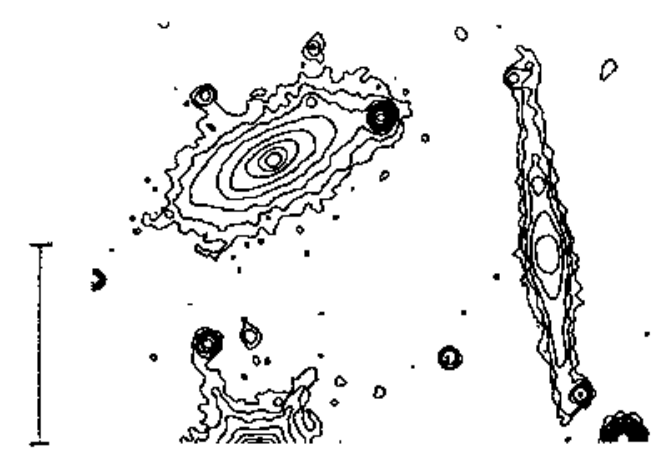

K 10

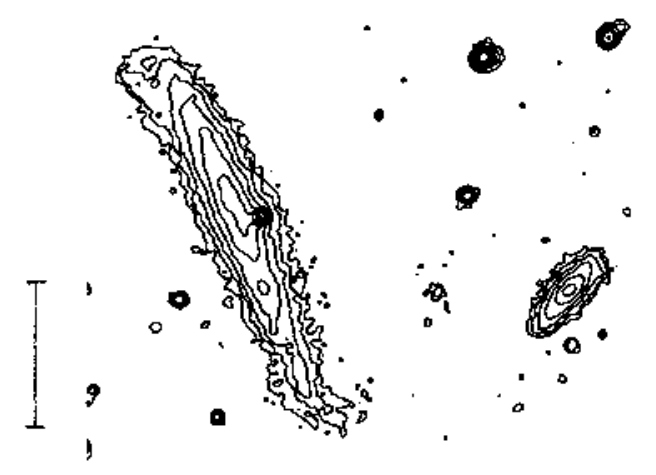

K 14

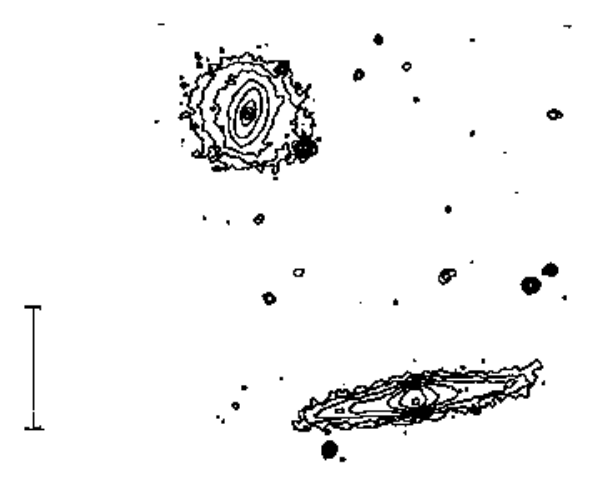

K 540

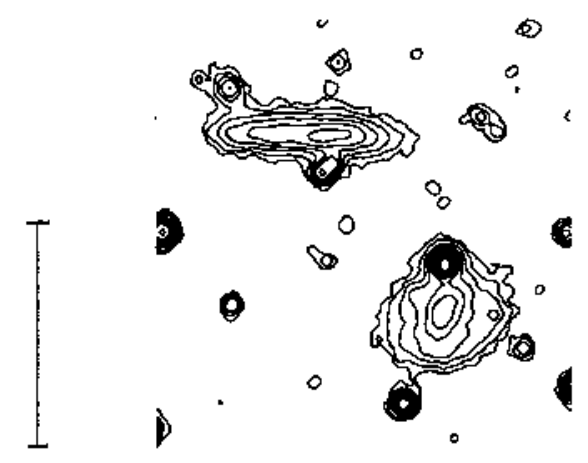

K 547

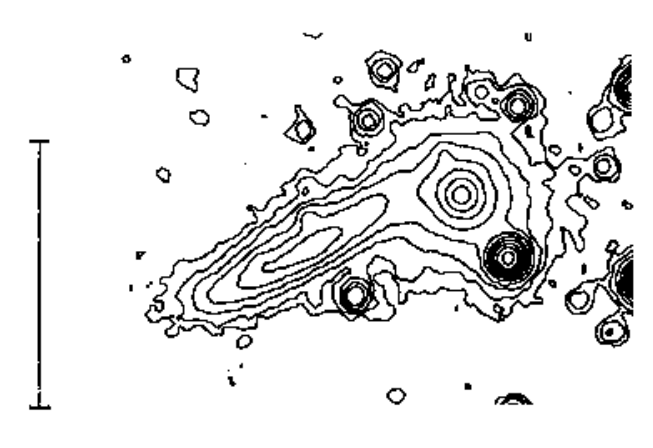

K 585

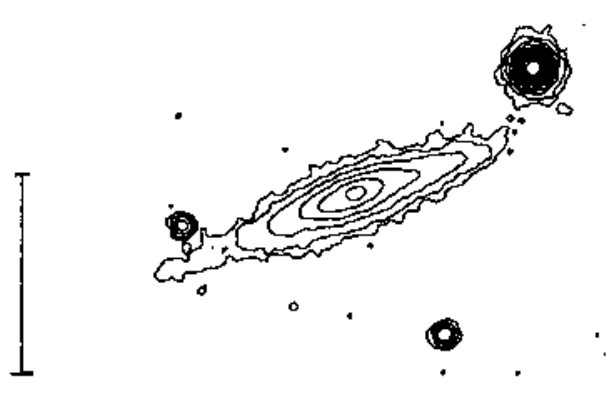

Fig. 1. continued 


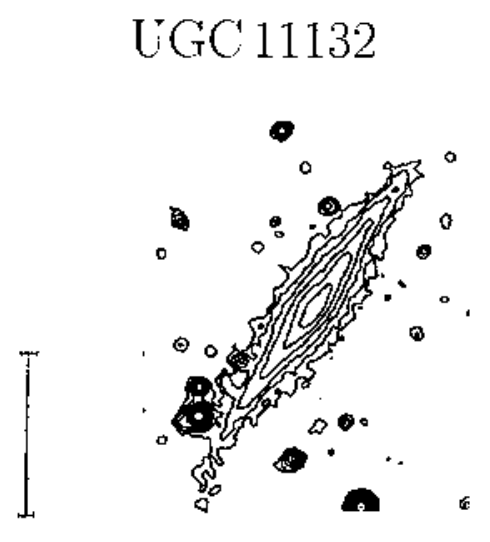

LGC 11230

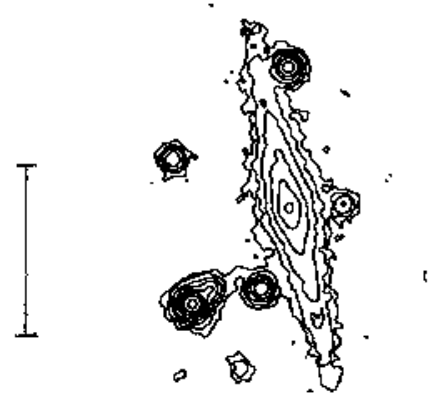

UGC 11301

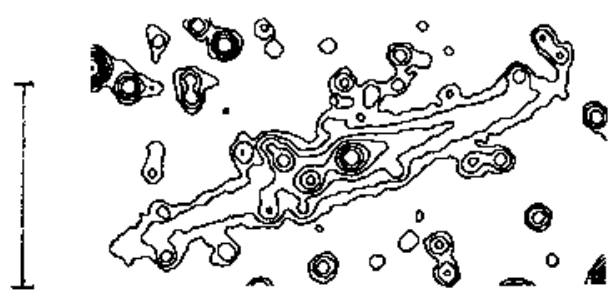

UGC 11838

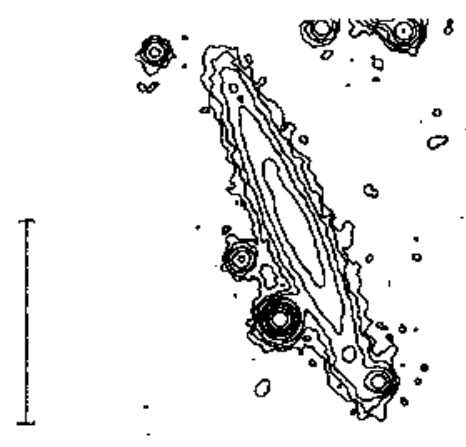

UGC 11841

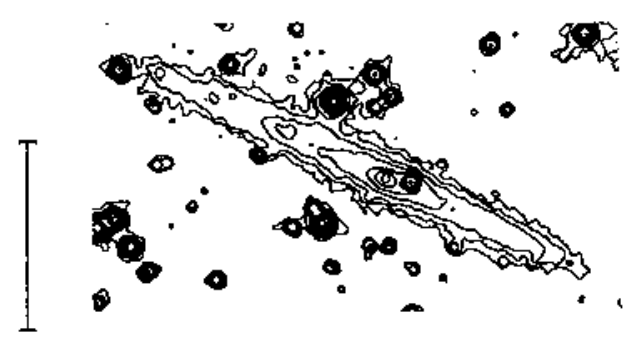

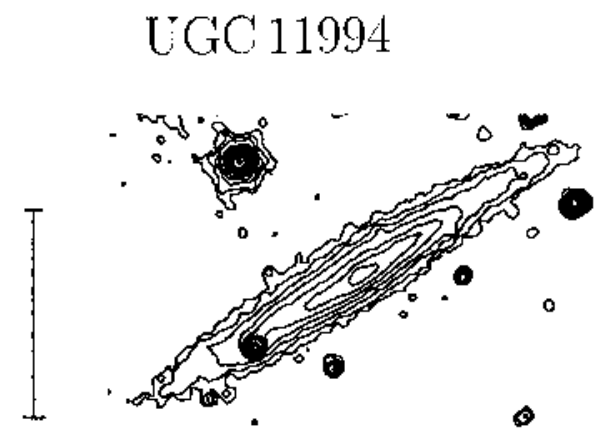

Fig. 1. continued 
background and its interpolation with a polynomial. Subtraction of the sky level has been made after having checked the smoothness of the polynomial in the regions occupied by the galaxies and the distribution of the residuals over the frame. The typical uncertainty of the background level is about $0.5 \%$. $V$ band isophotal contour images of the sample galaxies are presented in Fig. 1 (the surface brightnesses for the faintest contours are listed in the last column of Table 1).

Measuring the magnitudes of separate galaxies belonging to close interacting systems is not a trivial problem, since these galaxies are often partially superimposed on each other. Isophotal magnitudes (magnitudes contained within the given isophote) do not solve this problem. Indeed, our sample galaxies are often strongly overlapping (see Fig. 1) and relatively bright isophotal levels include several galaxies in many cases. If we try to use different isophotal levels for different galaxies, we have very inhomogeneous systems of magnitudes. To obtain uniform systems of magnitudes, we decided to determine the total asymptotic magnitudes and colours. Synthetic multi-aperture photometry was made for all galaxies and magnitude-aperture relations (growth curves) were constructed. The cosmetic defects and projected stars were excluded by masking. For the overlapping galaxies the size of the maximum aperture for each object was limited to the part of the galaxy free from any superposition. In some cases we excluded close companions by occulting them with rectangular masks. To derive asymptotic magnitudes and colours we used standard magnitude- and colour-aperture curves (de Vaucouleurs et al. 1991 (RC3); Buta et al. 1995). (For close interacting systems we verified our photometry by comparing the total magnitude of all summed individual components with observed magnitude within large aperture containing the whole system). Due to the large extrapolations used for many overlapping galaxies the resulting uncertainties of the measured $B_{\mathrm{t}}$ magnitudes may be as large as $0.15 \mathrm{mag}$ and for total colours of $0.05-0.10 \mathrm{mag}$.

The derived total magnitudes and colours are listed in Table 2 (without corrections for Galactic extinction and redshift).

\subsection{Photometry comparison}

\subsubsection{Aperture photometry}

Let us compare our results with multiaperture photoelectric photometry collected by Longo \& de Vaucouleurs $(1983,1985)$ (LdV). There are observations for four of our systems (Arp 71, Arp 112, Arp 284, Arp 286) in LdV. Figures $2 \mathrm{a}, \mathrm{b}$ show the differences between magnitudes and colours within the same apertures according to our and LdV data. The data are in good general agreement. The largest systematic deviations are encountered for Arp 286. For this object, the very old observations from de
Vaucouleurs 1959 and Tifft 1961 are affected by rather large systematic errors (Longo, private communication). Moreover, the data at $\log A=0.95$ (Tomov 1978, 1979) are also discrepant, which suggests possible errors. Excluding the data at $\log A=0.95$ and data for Arp 286, we find that the mean difference between our and LdV magnitudes amounts to $-0{ }^{\mathrm{m}} 03 \pm 0 .{ }^{\mathrm{m}} 02$ (s.e.m.) and for colours to $+0^{\mathrm{m}} \cdot 01 \pm 0^{\mathrm{m}} \cdot 01$.

\subsubsection{Schombert et al. (1990) photometry}

The results of $B$ and $V$ CCD surface photometry for Arp 242 and Arp 295 were published by Schombert et al. (1990) (SWS). Comparing our total $B_{\mathrm{t}}$ magnitudes with SWS measurements for the main galaxies plus tidal structures (14.61 and 14.54 for Arp 242A, B correspondingly; 14.48 and 14.71 for Arp 295NE, SW) we find an excellent agreement: the mean difference amounts to $B_{\mathrm{t}}(\mathrm{OHP})$ $-B(\mathrm{SWS})=+0{ }^{\mathrm{m}} 01 \pm 0{ }^{\mathrm{m}} 05$ (s.e.m.).

In the present work we determine $B-V$ colours of the members of Arp 242 and Arp 295, on average, of $0 \cdot 045 \pm 0.016$ redder as compared with SWS values. But this difference is unsignificant within colour errors in our and SWS works.

\subsubsection{Roth's (1994) photometry}

Roth (1994) published $V, I$ photometry of Arp $124 \mathrm{NE}$ (NGC 6361). We found that our $V$ and $I$ magnitudes are brighter by $0 .{ }^{\mathrm{m}} 15$ than in Roth (1994). In part, this difference may be due to our extrapolation to the total magnitudes while Roth used isophotal ones. Let us estimate the effect of extrapolation. According to Reshetnikov et al. (1993a, b) NGC 6361 reveals an exponentially decreasing surface brightness with scale length of about $15^{\prime \prime}$ in the $R_{\mathrm{c}}$ passband. Roth (1994) found a very similar value for the exponential scale length in the $I$ band: $h=13^{\prime \prime} 9$. Assuming pure exponential surface brightness distribution (see Fig. 2 in Reshetnikov et al. 1993b) we obtain a major axis effective radius for NGC 6361 of $R_{\mathrm{e}}$ $(I)=1.678 h=23^{\prime \prime} 3$. Now, Roth's $V$ and $I$ magnitudes were obtained within isophote $\mu_{I}=23.5$. Using the exponential scale-length and central surface brightness in the $I$ band found by Roth (1994), we obtain a major axis radius corresponding to this isophote: $R\left(\mu_{I}=23.5\right)=76^{\prime \prime}$. Then we find the abscissa from the standard growth curve (RC3, Buta et al. 1995): $\log R / R_{\mathrm{e}}=0.51$. This abscissa corresponds to the difference between total and isophotal magnitudes of $0{ }^{\mathrm{m}} 05-0 .{ }^{\mathrm{m}} 07$ for the late-type galaxy. Thus, the difference between our and Roth's magnitudes may be reduced to $0 .{ }^{\mathrm{m}} 08-0{ }^{\mathrm{m}} 10$ when the extrapolation effect is taken into account. Within our observational accuracy of $\left(0 .{ }^{\mathrm{m}} 10-0 .{ }^{\mathrm{m}} 15\right)$ and Roth's $\left(0{ }^{\mathrm{m}} 05\right)$ photometry this difference is unsignificant.

Our $V-I$ colour $-1{ }^{\mathrm{m}} 37$ - is close to that of Roth (1994) -1.36 . 
Table 2. Results

\begin{tabular}{|c|c|c|c|c|c|}
\hline Object & $\alpha(1950)$ & $\delta(1950)$ & $B_{\mathrm{t}}$ & $(B-V)_{\mathrm{t}}$ & $(V-I)_{\mathrm{t}}$ \\
\hline Arp 30N & 172214.2 & 621306 & 15.48 & 0.45 & 0.66 \\
\hline $\mathrm{S}$ & 172214.6 & 621240 & 14.76 & 0.63 & 0.86 \\
\hline Arp $71 \mathrm{~A}$ & 160253.1 & 175331 & 15.02 & 0.91 & 1.38 \\
\hline B & 160255.4 & 175332 & 17.0 & 0.76 & 1.16 \\
\hline $\mathrm{SE}$ & 160253.8 & 175156 & 14.73 & 0.93 & 1.32 \\
\hline NW & 160246.5 & 175436 & 15.30 & 0.97 & 1.26 \\
\hline 1 & 160247.7 & 175250 & 17.85 & 0.92 & 1.29 \\
\hline 2 & 160252.5 & 175510 & 18.2 & 0.35 & 0.74 \\
\hline 3 & 160245.0 & 175400 & 18.9 & 0.89 & 0.99 \\
\hline Arp 112 & 235854.0 & 310948 & 14.40 & 0.87 & 1.23 \\
\hline $\mathrm{E}$ & 235856.4 & 310952 & 16.46 & 0.64 & 0.98 \\
\hline W & 235852.7 & 310920 & 14.52 & 0.97 & 1.26 \\
\hline Arp $121 \mathrm{NE}$ & 005653.6 & -50352 & 14.49 & 1.00 & 1.23 \\
\hline SW & 005651.3 & -50425 & 14.19 & 1.05 & 1.41 \\
\hline Arp $124 \mathrm{NE}$ & 171803.4 & 603933 & 13.79 & 0.91 & 1.37 \\
\hline $\mathrm{SW}$ & 171753.7 & 603812 & 16.00 & 0.75 & 1.10 \\
\hline Arp $127 \mathrm{~N}$ & 003627.6 & $\begin{array}{lll}-9 & 16 & 39\end{array}$ & 13.68 & 1.00 & 1.26 \\
\hline $\mathrm{S}$ & 003628.3 & -9 1722 & 14.39 & 1.06 & 1.32 \\
\hline Arp 150A & 231658.2 & 091403 & 15.05 & 0.97 & 1.31 \\
\hline B & 231659.3 & 091342 & 15.74 & 0.79 & 1.30 \\
\hline $\mathrm{SE}$ & 231702.1 & 091316 & 15.90 & 0.92 & 1.39 \\
\hline SW & 231656.1 & 091314 & 16.76 & 0.80 & 1.16 \\
\hline Arp 208A & 164944.0 & 471847 & 15.09 & 0.41 & 0.60 \\
\hline B & 164945.7 & 471834 & 16.19 & 0.34 & 0.45 \\
\hline W & 164936.6 & 471825 & 16.78 & 0.62 & 0.80 \\
\hline Arp $242 \mathrm{~N}$ & 124344.2 & 310023 & 14.74 & 0.81 & 1.36 \\
\hline $\mathrm{S}$ & 124345.3 & 305951 & 14.47 & 0.82 & 1.29 \\
\hline Arp $278 \mathrm{NW}$ & 221708.6 & 290848 & 14.14 & 0.69 & 1.29 \\
\hline $\mathrm{SE}$ & 221711.3 & 290806 & 14.60 & 0.61 & 1.18 \\
\hline Arp $284 \mathrm{E}$ & 233348.5 & 015248 & $14.12 \mathrm{v}$ & & 0.73 \\
\hline W & 233341.2 & 015242 & $12.65 \mathrm{v}$ & & 0.91 \\
\hline Arp 286 & 141733.8 & 041318 & 13.09 & 0.86 & 1.16 \\
\hline Arp $295 \mathrm{NE}$ & 233926.6 & -35332 & 14.43 & 0.54 & 1.05 \\
\hline SW & 233913.1 & -35638 & 14.74 & 0.94 & 1.39 \\
\hline
\end{tabular}

\subsubsection{RC3 system of total magnitudes}

Let us compare our $B_{\mathrm{t}}$ magnitudes with RC3 (de Vaucouleurs et al. 1991) system of total magnitudes. Among our sample objects we found 19 galaxies with $B_{\mathrm{t}}$ magnitudes in RC3. Figure 3 gives the relationship between our - $B_{\mathrm{t}}(\mathrm{OHP})$ - and RC3 magnitudes - $B_{\mathrm{t}}(\mathrm{RC} 3)$. The agreement between the two systems of magnitudes is good. The mean difference $B_{\mathrm{t}}(\mathrm{OHP})-B_{\mathrm{t}}(\mathrm{RC} 3)$ is 0. $00 \pm 0 .{ }^{\mathrm{m}} 06$ (s.e.m).

\subsection{5. $R_{\mathrm{c}}$ photometry}

There are total magnitudes in the Cousins $R_{\mathrm{c}}$ system for ten of our sample galaxies in Reshetnikov et al. (1993b). The $R_{\mathrm{c}}$ passband is bracketed by the $V$ and $I$ bands and so we can expect a correlation of $R_{\mathrm{c}}$ magnitudes with linear combination of $V$ and $I$ values. Indeed, excluding one strongly deviating galaxy (Arp 284B), we found very good correlation: $R_{\mathrm{c}}=V-0.50(V-I)+0.19$ (Fig. 4). The galaxies follow this empirical relationship with standard deviation of 0.11 only.

Therefore, one can conclude from our estimates and comparisons with published results that the accuracy of our photometry is not worse than 0.15 .

\section{Comments on individual objects}

In this section we will briefly discuss the identification of some galaxies listed in Table 2 with Fig. 1. 
Table 2. continued

\begin{tabular}{|c|c|c|c|c|c|}
\hline Object & $\alpha(1950)$ & $\delta(1950)$ & $B_{\mathrm{t}}$ & $(B-V)_{\mathrm{t}}$ & $(V-I)_{\mathrm{t}}$ \\
\hline VV 426NW & 174433.6 & 304323 & 14.40 & 0.56 & 0.98 \\
\hline $\mathrm{SE}$ & 174437.4 & 304301 & 15.88 & 0.40 & 0.79 \\
\hline VV 489N & 161043.2 & 282636 & 17.4 & 0.53 & 0.94 \\
\hline $\mathrm{S}$ & 161042.7 & 282456 & 14.77 & 0.54 & 0.98 \\
\hline 1 & 161043.4 & 282508 & 16.9 & 0.81 & 1.25 \\
\hline 2 & 161043.2 & 282415 & 17.5 & 0.37 & 0.66 \\
\hline 3 & 161041.5 & 282430 & 19.9 & 0.94 & 1.29 \\
\hline 4 & 161040.5 & 282434 & 19.9 & 0.98 & 1.10 \\
\hline VV 490N & 230745.7 & -85739 & 14.68 & 1.13 & 1.42 \\
\hline $\mathrm{S}$ & 230744.7 & -85757 & 15.75 & 1.05 & 1.21 \\
\hline VV 679A & 182203.4 & 663523 & 14.24 & 0.60 & 1.06 \\
\hline B & 182205.7 & 663543 & 15.5 & 0.39 & 0.72 \\
\hline $\mathrm{SE}$ & $\begin{array}{lll}18 & 22 & 20.7\end{array}$ & 663225 & 16.9 & 0.93 & 1.14 \\
\hline VV $773 \mathrm{E}$ & $2025 \quad 14.7$ & 103520 & 14.21 & -0.13 & 0.31 \\
\hline W & 202511.8 & 103531 & 15.94 & 0.06 & 0.47 \\
\hline $\mathrm{K} 3 \mathrm{E}$ & 000754 . & 284400 & 14.72 & 0.86 & 1.34 \\
\hline W & 000748 . & 284333 & 16.41 & 1.03 & 1.56 \\
\hline $\mathrm{K} 10 \mathrm{E}$ & 002430 . & 111800 & 13.78 & 0.54 & 0.96 \\
\hline $\mathrm{W}$ & 002420 . & 111724 & 15.75 & 0.51 & 0.83 \\
\hline $\mathrm{K} 14 \mathrm{NE}$ & 003706 . & 084300 & 14.80 & 0.76 & 1.17 \\
\hline SW & 003700. & 084042 & 15.00 & 0.92 & 1.48 \\
\hline K 540NE & 192700 & 651300. & 15.53 & 0.42 & 0.76 \\
\hline SW & 192656 . & $\begin{array}{lll}65 & 12 & 10\end{array}$ & 15.65 & 0.52 & 0.95 \\
\hline K 547E & 203218 . & 074800 & 15.74 & 0.95 & 1.40 \\
\hline W & 203215.8 & 074748 & 15.67 & 0.93 & 1.24 \\
\hline K 585 & 233048 . & 205700 & 15.33 & 0.69 & 1.06 \\
\hline UGC 11132 & 180745.9 & 384702 & 14.85 & 0.81 & 1.26 \\
\hline UGC 11230 & 182356.3 & 651638 & 15.60 & 0.83 & 1.34 \\
\hline UGC 11301 & 183542.2 & 172922 & 15.10 & 0.89 & 1.36 \\
\hline UGC 11838 & 215020.5 & 280413 & 15.21 & 0.60 & 0.98 \\
\hline UGC 11841 & 215042.3 & 384207 & 16.24 & 1.30 & 1.61 \\
\hline UGC 11859 & 215534.0 & 004613 & 15.49 & 0.79 & 1.26 \\
\hline UGC 11994 & 221838.1 & 330227 & 14.46 & 0.99 & 1.36 \\
\hline
\end{tabular}

\section{Arp 71}

This interacting system is placed in the central part of the cluster Abell 2151. The object marked by letter A in Table 2 is the large edge-on galaxy near the center of the frame. Letter B denotes the small companion from the East side of the galaxy A. Arp 71SE is the large galaxy in the SE corner of the figure; Arp 71NW locates in the NW corner; numbers 1-3 mark small galaxies in the field.

\section{Arp 112}

The galaxy with extended tail in the center of figure is Arp 112; it has East and West companions denoted by the corresponding letters.

\section{Arp 150}

The North component of the close pair of galaxies connected by a bridge is marked as A, the South component as B. The galaxies in the South-East and South-West corners of the figure are indicated by SE and SW in the Table 2.

\section{Arp 208}

SE component of the close pair of galaxies in the East side of the figure is denoted as A, the NW component - as B. The edge-on galaxy in the West side is marked as $\mathrm{W}$.

\section{VV 489}

The peculiar object in the South part of the figure is marked by the letter S, the North galaxy - as N. Individual condensations (galaxies, probably) in VV 489S are indicated by the numbers 1-4.

\section{VV 679}

The large edge-on galaxy near the bright star is denoted as A, while the letter B denotes the small compact companion projected to the North-East part of the galaxy A. 

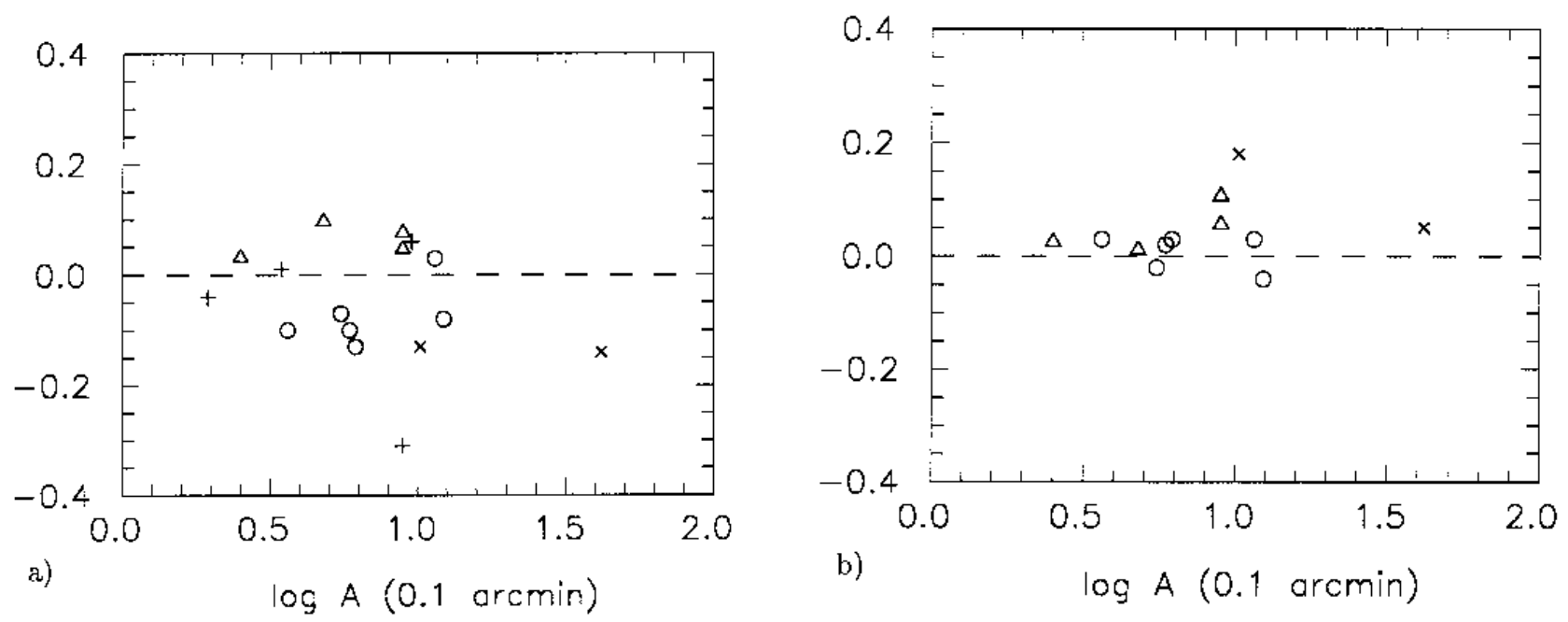

Fig. 2. a) Difference between our $B$ magnitudes ( $V$ for Arp 284) and those of LdV as a function of aperture; b) a similar plot for the $B-V$ colour index. Circles represent the data for Arp 71, triangles - Arp 112, right crosses - Arp 284, inclined crosses - Arp 286

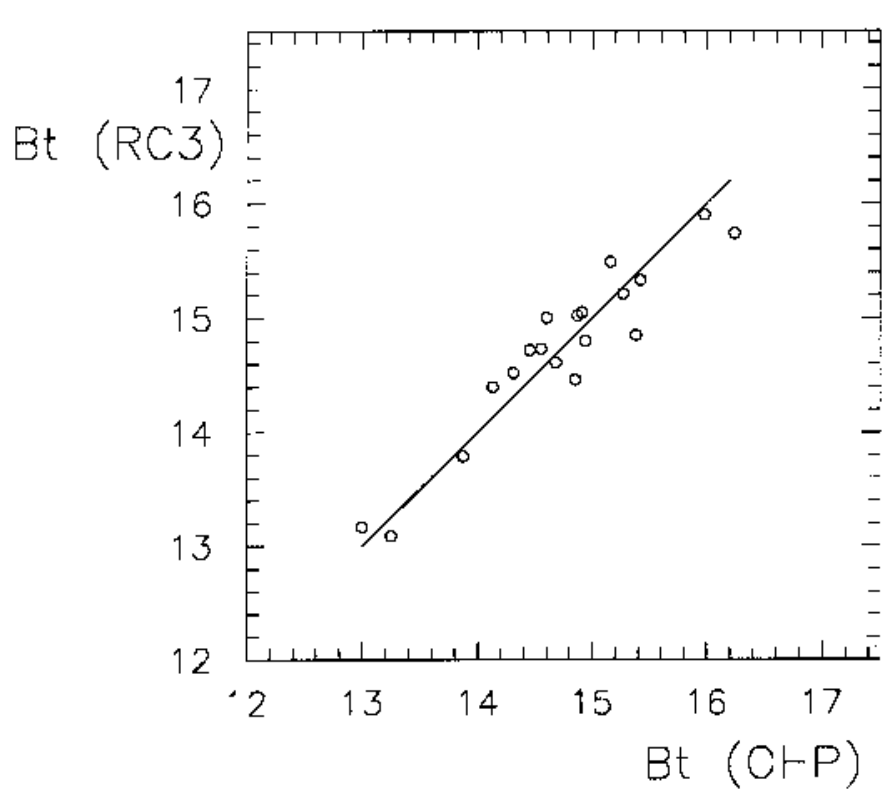

Fig. 3. Comparison of our and RC3 total magnitudes. Solid line represents the dependence $B_{\mathrm{t}}(\mathrm{RC} 3)=B_{\mathrm{t}}(\mathrm{OHP})$

The faint galaxy in the South-East corner is marked as SE.

\section{Conclusions}

In this article we presented the results of $B, V, I$ CCD surface photometry for the members of 24 interacting systems (containing at lEast one nearly edge-on disk galaxy) and for 7 non-interacting edge-on spirals from the UGC

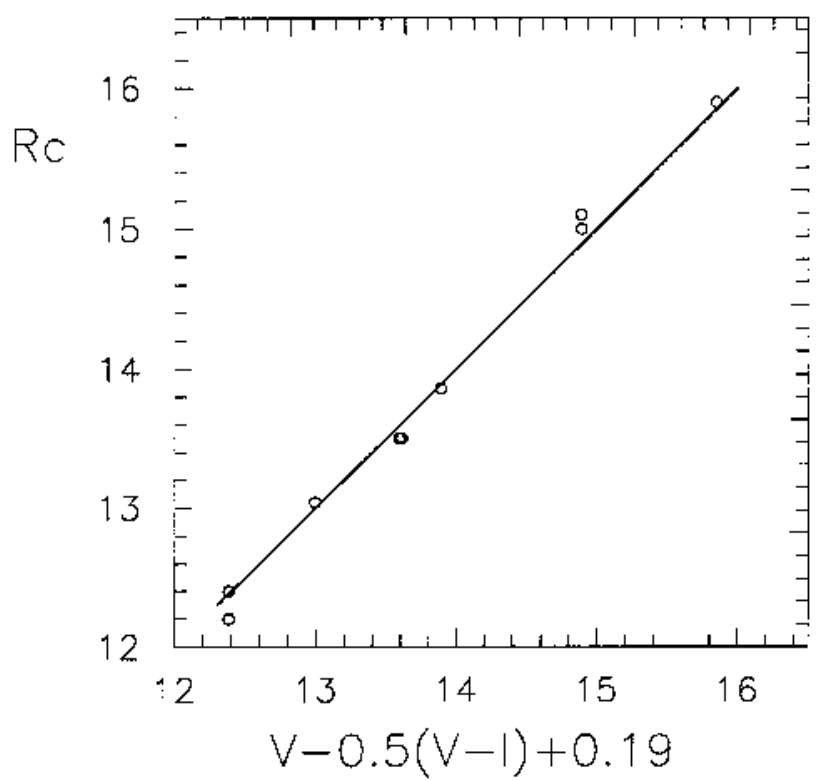

Fig. 4. Correlation found between $R_{\mathrm{c}}$ magnitudes from Reshetnikov et al. (1993b) and a linear combination of $V$ and $I$ values taken from the present work. The solid line has a slope of unity

catalog (Nilson 1973). We plan to use this observations for the study of the efficiency of tidal thickening of the galactic disks.

Acknowledgements. We thank G. Longo for refereeing thoroughly this article. V.R. is grateful to CNRS and French Minister for Research (MRT) for financial support during his stay in Meudon. V.R. acknowledges also the support of the ESO 
C\&EE Programme (grant $N$ B-04-009). We have made use of the Lyon-Meudon Extragalactic Database (LEDA) supplied by the LEDA team at the CRAL-Observatoire de Lyon (France).

\section{References}

Arp H.C., 1966, "Atlas of Peculiar Galaxies", Pasadena Binney J.J., Tremaine S.D., 1987, "Galactic Dynamics". Princeton University Press

Buta R., Corwin H.G., de Vaucouleurs G., de Vaucouleurs A., Longo G., 1995, AJ 109, 517

Chevalier C., Ilovaisky S.A., 1991, A\&AS 90, 225

Combes F., Debbasch F., Friedli D., Pfenniger D., 1990, A\&A 233,82

de Vaucouleurs G., 1959, Lowell Obs. Bull. 4, No. 97

de Vaucouleurs G., de Vaucouleurs A., Corwin H.G., et al., 1991, "Third Reference Catalogue of Bright Galaxies" (RC3). Springer-Verlag

Elmegreen D.M., Elmegreen B.G., 1982, MNRAS 201, 1021

Gerin M., Combes F., Athanassoula E., 1990, A\&A 230, 37

Karachentsev I.D., 1972, Comm. Spec. Ap. Obs. USSR 7, 3

Karachentsev I.D., 1987, "Binary Galaxies", Moscow

Landolt A.U., 1983, AJ 88, 439

Longo G., de Vaucouleurs A., 1983, University of Texas
Monographs in Astron. No. 3 (LdV)

Longo G., de Vaucouleurs A., 1985, University of Texas Monographs in Astron. No. 3A ( LdV)

Nilson P., 1973, "Uppsala General Catalogue of Galaxies", Uppsala

Noguchi M., 1987, MNRAS 221, 41

Quinn P.J., Hernquist L., Fullagar D.P., 1993, ApJ 403, 74

Raha N., Sellwood J.A., James R.A., Kahn F.D., 1991, Nat 352,411

Reshetnikov V.P., Hagen-Thorn V.A., Yakovleva V.A., 1993a, A\&A 278, 351

Reshetnikov V.P., Hagen-Thorn V.A., Yakovleva V.A., 1993b, A\&AS 99, 257

Roth J., 1994, AJ 108, 862

Schombert J.M., Wallin J.F., Struck-Marcell C., 1990, AJ 99, 497

Smith P.S., Jannuzi B.T., Elston R., 1991, ApJS 77, 67

Thompson L.A., 1981, ApJ 244, L43

Tifft W.G., 1961, A\&A 66, 390

Tomov A.H., 1978, Astron. Zh. 55, 944

Tomov A.H., 1979, Astron. Zh. 56, 949

Toth G., Ostriker J.P., 1992, ApJ 389, 5

Vorontsov-Velyaminov B.A., 1977, A\&AS 28, 1 